\title{
Exact Controllability to the Trajectories for Parabolic PDEs with Nonlocal Nonlinearities
}

\author{
Enrique FERNÁNDEZ-CARA, J. LÍMACO, \\ Dany NinA-HuAMAN ${ }^{\ddagger}$ and Miguel R. NúÑEZ-CHÁVEZ ${ }^{\S}$
}

\begin{abstract}
This paper deals with the analysis of the internal control of a parabolic PDE with nonlinear diffusion, nonlocal in space. In our main result, we prove the local exact controllability to the trajectories with distributed controls, locally supported in space. The main ingredients of the proof are a compactness-uniqueness argument and Kakutani's Fixed-Point Theorem in a suitable functional setting. Some possible extensions and open problems concerning other nonlocal systems are presented.
\end{abstract}

Mathematics Subject Classification: 35B37; 93C20.

Key words and phrases: Nonlinear parabolic PDE, exact controllability to the trajectories, internal control.

\section{Introduction}

We analyze the problem of exact controllability to the trajectories with distributed controls for a parabolic system with nonlocal in space and nonlinear diffusion.

Let $\Omega \subset \mathbb{R}^{N}$ be a non-empty bounded connected open set, with regular boundary $\partial \Omega(N \geq 1$ is an integer). We fix $T>0$ and set $Q:=\Omega \times(0, T)$ and $\Sigma:=\partial \Omega \times(0, T)$. In the sequel, we denote by $(\cdot, \cdot)$ and $\|\cdot\|$ respectively the $L^{2}$ scalar product and norm in $\Omega$; the symbol $C$ is used to design a generic positive constant.

Let $\omega \subset \Omega$ be a non-empty open set. We deal with the exact controllability

*Dpto. EDAN and IMUS, Univ. Sevilla, Aptdo. 1160, 41080 Sevilla, Spain. Partially supported by MINECO Spain), grant MTM2016-76990-P, cara@us .es.

$\dagger$ IME, Univ. Federal Fluminense, RJ, Brasil, jl imaco@vm. uff.br.

$\ddagger$ IME, Univ. Federal Fluminense , RJ, Brasil, danynina3003@gmail . com.

$\S$ IME, Univ. Federal Fluminense, RJ, Brasil, mi guel nunez 9813@gmai l . com. 
to the trajectories for the nonlinear system

$$
\begin{cases}y_{t}-a\left(\int_{\Omega} y d x^{\prime}\right) \Delta y=v 1_{\omega} & \text { in } Q, \\ y(x, t)=0 & \text { on } \Sigma, \\ y(x, 0)=y_{0}(x) & \text { in } \Omega,\end{cases}
$$

where $v$ is a control and $y$ is an associated state. Here, it will be assumed that the real function $a=a(r)$ satisfies

$$
a \in C^{2}(\mathbb{R}), \quad 0<a_{0} \leq a(r) \leq a_{1} \text { and }\left|a^{\prime}(r)\right|+\left|a^{\prime \prime}(r)\right| \leq M \forall r \in \mathbb{R} .
$$

Note that, if $y_{0} \in H^{2}(\Omega) \cap H_{0}^{1}(\Omega), v \in L^{2}(\omega \times(0, T))$ and $v_{t} \in L^{2}(\omega \times(0, T))$, then (1.1) possesses exactly one strong solution among other things satisfying

$$
y, y_{t} \in L^{\infty}\left(0, T ; H_{0}^{1}(\Omega)\right) \text { and } y_{t t} \in L^{2}\left(0, T ; H^{-1}(\Omega)\right) ;
$$

see for instance [3].

Let us consider a trajectory $\bar{y}=\bar{y}(x, t)$, that is, a solution to

$$
\begin{cases}\bar{y}_{t}-a\left(\int_{\Omega} \bar{y} d x^{\prime}\right) \Delta \bar{y}=0 & \text { in } \quad Q, \\ \bar{y}(x, t)=0 & \text { on } \Sigma, \\ \bar{y}(x, 0)=\bar{y}_{0}(x) & \text { in } \Omega,\end{cases}
$$

where

$$
\bar{y}_{0} \in H^{2}(\Omega) \cap H_{0}^{1}(\Omega) .
$$

Definition 1.1. It will be said that (1.1) is locally exactly-controllable to the trajectory $\bar{y}$ at time $T$ if there exists $\epsilon>0$ with the following property: if $y_{0} \in H^{2}(\Omega) \cap$ $H_{0}^{1}(\Omega)$ and

$$
\left\|\bar{y}_{0}-y_{0}\right\|_{H^{2}} \leq \epsilon
$$

then there exist controls $v \in L^{2}(\omega \times(0, T))$ and associated states $y$ such that

$$
y(x, T)=\bar{y}(x, T) \text { in } \Omega .
$$

The nonlocal term in (1.1) has important motivations in physics and biology. To this respect, let us recall several examples of real world models where it appears naturally:

- In the case of migration of populations, for instance the bacteria in a container, we may have a diffusion coefficient at time $t$ that depends on the total population.

- In the context of reaction-diffusion systems, it is also frequent to find terms of this kind; the particular case

$$
a(\langle\ell, y(\cdot, t)\rangle)
$$

where $a=a(s)$ is as above and $\ell$ is a continuous linear form on $L^{2}(\Omega)$, has been investigated for instance by Chang and Chipot [2]. We refer to this paper for more details. 
Recently, important progress has been made in the controllability analysis of linear and semi-linear parabolic equations and systems. We refer to the works $[4,5,6,9,13]$ and the references therein. In particular, the null controllability of linear parabolic (and hyperbolic) PDEs containing nonlocal in space terms has been the objective of [6]. In the present paper, the main novelty is that we deal with the exact controllability to regular trajectories for systems of the kind (1.1).

Our main result is the following:

Theorem 1.1. Under the assumptions (1.2), (1.3) and (1.4), the nonlinear system (1.1) is locally exactly controllable to the trajectory $\bar{y}$ at any time $T>0$.

Note that, if we set $y=z+\bar{y}$ and $y_{0}=z_{0}+\bar{y}_{0}$, we obtain

$$
\begin{cases}z_{t}-a\left(\int_{\Omega}(z+\bar{y}) d x^{\prime}\right) \Delta z-m(z) \Delta \bar{y}=v 1_{\omega} & \text { in } Q \\ z(x, t)=0 & \text { on } \Sigma \\ z(x, 0)=z_{0}(x) & \text { in } \Omega\end{cases}
$$

where

$$
m(z):=a\left(\int_{\Omega}(z+\bar{y}) d x^{\prime}\right)-a\left(\int_{\Omega} \bar{y} d x^{\prime}\right) .
$$

Thus, the local exact controllability of the solution to (1.1) to $\bar{y}$ is equivalent to the null controllability of the solution to (1.6).

The null controllability problem for (1.6) can be formulated as a fixed-point inclusion in the Banach space $Z$. Consequently, a good strategy for the proof of Theorem 1.1 seems to be to check that, if $z_{0}$ is small enough, Kakutani's FixedPoint Theorem can be applied. However, this does not seem easy: it is not clear whether the closed-graph assumption is satisfied in this context.

For this reason, we will first see that (1.6) is locally approximately controllable to zero. In other words, we will prove that there exists $\epsilon>0$ such that, if $z_{0} \in H^{2}(\Omega) \cap H_{0}^{1}(\Omega)$ and

$$
\left\|z_{0}\right\|_{H^{2}} \leq \epsilon
$$

then for all small $\delta>0$ there exist controls $v_{\delta}$ uniformly bounded in $L^{2}(\omega \times$ $(0, T))$ and associated states $z_{\delta}$ satisfying

$$
\left\|z_{\delta}(\cdot, T)\right\| \leq \delta .
$$

To this purpose, we will apply Kakutani's Fixed-Point Theorem to an appropriate formulation of the approximate null controllability problem for (1.6).

More precisely, in a first step, we will consider for each $w \in L^{2}(Q)$ with $w_{t} \in$ $L^{\infty}\left(0, T, L^{2}(\Omega)\right)$ the linearized system

$$
\begin{cases}z_{t}-\alpha_{w}(t) \Delta z+\beta_{w}(t)\left(\int_{\Omega} z d x^{\prime}\right) \Delta \bar{y}=v 1_{\omega} & \text { in } Q, \\ z(x, t)=0 & \text { on } \Sigma, \\ z(x, 0)=z_{0}(x) & \text { in } \Omega,\end{cases}
$$


where

$$
\alpha_{w}(t):=a\left(\int_{\Omega}(w+\bar{y}) d x^{\prime}\right)
$$

and

$$
\beta_{w}(t):= \begin{cases}-\frac{a\left(\int_{\Omega}(w+\bar{y}) d x^{\prime}\right)-a\left(\int_{\Omega} \bar{y} d x^{\prime}\right)}{\int_{\Omega} w d x^{\prime}} & \text { if } \int_{\Omega} w d x^{\prime} \neq 0, \\ -a^{\prime}\left(\int_{\Omega} \bar{y} d x^{\prime}\right) & \text { otherwise. }\end{cases}
$$

The adjoint of (1.7) is

$$
\begin{cases}-\varphi_{t}-\alpha_{w}(t) \Delta \varphi+\beta_{w}(t) \int_{\Omega} \Delta \bar{y}\left(x^{\prime}, t\right) \varphi\left(x^{\prime}, t\right) d x^{\prime}=0 & \text { in } Q, \\ \varphi(x, t)=0 & \text { on } \Sigma, \\ \varphi(x, T)=\varphi_{T}(x) & \text { in } \Omega,\end{cases}
$$

where $\varphi_{T} \in L^{2}(\Omega)$.

We will prove an observability estimate for the solutions to (1.8) whence we will immediately deduce the approximate controllability of (1.7). To this end, we will argue as in [6], employing a compactness-uniqueness technique. Then, a classical fixed point argument will ensure the same property for (1.6). It is important to observe that the occurrence of the nonlocal term in the PDE in (1.8) is a serious obstacle to the obtention of Carleman estimates. Here, as in [6], we have to present a more subtle proof of observability.

After this, in a final step, we will take limit as $\delta \rightarrow 0$ and the desired local null controllability of (1.6) will be obtained.

The paper is organized as follows.

Section 2 is devoted to prove the observability inequality for the solutions to (1.8) and then the approximate null controllability of (1.7).

In Section 3, we prove Theorem 1.1.

Section 4 deals with some additional comments and results.

\section{Some Technical Results}

\subsection{Preliminar lemmas}

We will need some (well known) results from Fursikov and Imanuvilov [9]; see also [10]. Also, it will be convenient to introduce a new non-empty open set $\omega_{0}$, with $\omega_{0} \Subset \omega$. The following technical result, due to Fursikov and Imanuvilov [9], is fundamental:

Lemma 2.1. There exists a function $\sigma_{0} \in C^{2}(\bar{\Omega})$ satisfying:

$$
\left\{\begin{array}{l}
\sigma_{0}(x)>0 \forall x \in \Omega, \sigma_{0}(x)=0 \forall x \in \partial \Omega \text { and } \\
\left|\nabla \sigma_{0}(x)\right|>0 \forall x \in \bar{\Omega} \backslash \omega_{0} .
\end{array}\right.
$$


Let us introduce the functions

$$
\sigma(x, t)=\frac{e^{4 \lambda\left\|\sigma_{0}\right\|_{\infty}}-e^{\lambda\left(2\left\|\sigma_{0}\right\|_{\infty}+\sigma_{0}(x)\right)}}{\ell(t)}, \quad \xi(x, t)=\frac{e^{\lambda\left(2\left\|\sigma_{0}\right\|_{\infty}+\sigma_{0}(x)\right)}}{\ell(t)},
$$

where

$$
\ell(t)=\left\{\begin{array}{l}
\frac{T^{2}}{4}, 0 \leq t \leq T / 2, \\
t(T-t), T / 2 \leq t \leq T
\end{array}\right.
$$

and $\lambda>0$.

We will denote by $\lambda_{1}, \lambda_{2}, \ldots$, (respectively $\phi_{1}, \phi_{2}, \ldots$ ) the eigenvalues (respectively the unit $L^{2}$ norm eigenfunctions) of the Dirichlet Laplacian in $\Omega$. Recall that $0<\lambda_{1}<\lambda_{2} \leq \lambda_{3} \leq \ldots, \lambda_{m} \sim m^{2 / N}$ as $m \rightarrow+\infty$ and $\phi_{1}>0$ in $\Omega$.

In the sequel, we will need the Banach space

$$
Z:=\left\{w \in L^{2}(Q): w_{t} \in L^{\infty}\left(0, T, L^{2}(\Omega)\right)\right\} .
$$

Lemma 2.2. Let the assumptions (1.2) be satisfied. Let $w \in Z$ be given and let us set $b(s):=a\left(\int_{\Omega} w\left(x^{\prime}, s\right) d x^{\prime}\right)$. There exist $R_{0}$ and $C_{0}$, depending on $\Omega, \omega, T, a_{0}, a_{1}, M$ and $\|w\|_{Z}$, such that, for all $f \in L^{2}(\Omega)$, one has:

$$
\begin{aligned}
& \sum_{j \geq 1} e^{-2 R_{0}} \sqrt{\lambda_{j}}\left|\left(f, \phi_{j}\right)\right|^{2} \\
& \quad \leq C_{0} \iint_{\omega \times(0, T)} e^{-2 s \sigma} \xi^{3}\left|\sum_{j \geq 1}\left(f, \phi_{j}\right) e^{-\lambda_{j} \int_{t}^{T} b(s) d s} \phi_{j}(x)\right|^{2} d x d t .
\end{aligned}
$$

The proof uses arguments close to those in [6]. For completeness, it is given in the Appendix (see Section 5).

\subsection{Some Observability Inequalities}

For further use, we introduce the following notation:

$$
I(\varphi):=\iint_{Q} e^{-2 s \sigma}\left[(s \xi)^{-1}\left(\left|\varphi_{t}\right|^{2}+|\Delta \varphi|^{2}\right)+(s \xi)|\nabla \varphi|^{2}+(s \xi)^{3}|\varphi|^{2}\right] d x d t .
$$

In the next result, we present some observability estimates for the solutions to the adjoint systems (1.8) that play a crucial role in the proof of Theorem 1.1.

Proposition 2.1. Let the assumptions (1.2) be satisfied. There exist $s$ and $C>0$, only depending on $\Omega, \omega, T, a_{0}, a_{1}, M$ and $K$, such that, for any $\varphi^{T} \in L^{2}(\Omega)$ and any $w \in Z$ with $\|w\|_{Z} \leq K$, the corresponding solution to (1.8) satisfies

$$
\|\varphi(\cdot, 0)\|^{2} \leq C \iint_{\omega \times(0, T)} e^{-2 s \sigma} \xi^{3}|\varphi|^{2} d x d t
$$

and

$$
I(\varphi) \leq C \iint_{\omega \times(0, T)} e^{-2 s \sigma} \xi^{3}|\varphi|^{2} d x d t
$$


Proof. Let us first prove (2.10). To this end, we will argue as in the proof of Theorem 3 in [6].

For any $\varphi^{T} \in L^{2}(\Omega)$, let us denote by $\varphi$ the corresponding solution to (1.8) and let us write

$$
\varphi=p+\zeta,
$$

where $p$ is the unique solution to

$$
\begin{cases}-p_{t}-\alpha_{w}(t) \Delta p=0 & \text { in } \quad Q \\ p(x, t)=0 & \text { on } \Sigma \\ p(x, T)=\varphi_{T}(x), & \text { in } \Omega\end{cases}
$$

Obviously, one has:

$$
\begin{cases}-\zeta_{t}-\alpha_{w}(t) \Delta \zeta+\beta_{w}(t) B(\zeta)(t)=-\beta_{w}(t) B(p)(t) & \text { in } Q, \\ \zeta(x, t)=0 & \text { on } \Sigma, \\ \zeta(x, T)=0, & \text { in } \Omega,\end{cases}
$$

where we have introduced the notation

$$
B(\zeta)(t):=\int_{\Omega} \Delta \bar{y}\left(x^{\prime}, t\right) \zeta\left(x^{\prime}, t\right) d x^{\prime} .
$$

It is well known that, for any $s>0$ such that the mapping

$$
\varphi^{T} \mapsto\left\|\varphi^{T}\right\|_{\omega}:=\left(\iint_{\omega \times(0, T)} e^{-2 s \sigma} \xi^{3}|p|^{2} d x d t\right)^{1 / 2}
$$

is a norm in $L^{2}(\Omega)$. Also, using standard global Carleman estimates in (2.12), it can be deduced that there exists $s\left(\Omega, \omega, T, a_{0}, a_{1}, M,\|w\|_{Z}\right)$ and $C\left(\Omega, \omega, a_{0}, a_{1}, M,\|w\|_{Z}\right)$ such that

$$
I(p) \leq C\left\|\varphi^{T}\right\|_{\omega}^{2}
$$

for all $\varphi^{T} \in L^{2}(\Omega)$; see [9]. On the other hand, since

$$
p(x, t)=\sum_{j \geq 1} e^{-\lambda_{j} \int_{t}^{T} \alpha_{w}(s) d s}\left(\varphi^{T}, \phi_{j}\right) \phi_{j}(x),
$$

we see from Lemma 2.2 that

$$
\sum_{j \geq 1} e^{-2 R_{0} \sqrt{\lambda_{j}}}\left|\left(\varphi^{T}, \phi_{j}\right)\right|^{2} \leq C_{0}\left\|\varphi^{T}\right\|_{\omega}^{2} .
$$

The inequality (2.10) will be a consequence of the following two estimates, that must hold for any $\varphi^{T} \in L^{2}(\Omega)$ and any $w \in Z$ with $\|w\|_{Z} \leq K$ :

$$
\left\|\varphi^{T}\right\|_{\omega}^{2} \leq C \iint_{\omega \times(0, T)} e^{-2 s \sigma} \xi^{3}|\varphi|^{2} d x d t \quad \forall \varphi^{T} \in L^{2}(\Omega)
$$


and

$$
\|\varphi(\cdot, 0)\|^{2} \leq C\left\|\varphi^{T}\right\|_{\omega}^{2} \quad \forall \varphi^{T} \in L^{2}(\Omega)
$$

(here, $C$ can depend on $\Omega, \omega, T, a_{0}, a_{1}, M$ and $K$ ).

Let us give their proofs.

Proof of (2.16). Here, we argue by contradiction and we employ a compactnessunique method.

Let us assume that (2.16) does not hold. Then, for every $n \geq 1$, we can find functions $\varphi_{n}^{T} \in L^{2}(\Omega)$ and $w_{n} \in Z$ such that $\left\|w_{n}\right\|_{Z} \leq K$ and

$$
1=\left\|\varphi_{n}^{T}\right\|_{\omega}^{2}>n \iint_{\omega \times(0, T)} e^{-2 s \sigma} \xi^{3}\left|\varphi_{n}\right|^{2} d x d t,
$$

where $\varphi_{n}$ is the solution to (1.8) associated to $\varphi_{n}^{T}$ and $w_{n}$.

Let us denote by $p_{n}$ (resp. $\zeta_{n}$ ) the solution to (2.12) corresponding to $\varphi^{T}=$ $\varphi_{n}^{T}$ and $w=w_{n}$ (resp. the solution to (2.13) for $p=p_{n}$ and $w=w_{n}$ ). Let us see that, at least for a subsequence, one must have

$$
\zeta_{n} \rightarrow 0 \text { strongly in } L^{2}(Q) .
$$

This will lead to a contradiction, since we will then have

$$
1=\left\|\varphi_{n}^{T}\right\|_{\omega}^{2} \leq 2 \iint_{\omega \times(0, T)} e^{-2 s \sigma} \xi^{3}\left|\varphi_{n}\right|^{2} d x d t+2 \iint_{\omega \times(0, T)} e^{-2 s \sigma} \xi^{3}\left|\zeta_{n}\right|^{2} d x d t
$$

and both terms in the right-hand side go to zero.

In order to prove (2.19), let us first check that

$$
\left\|\beta_{w_{n}}(\cdot) \int_{\Omega} \Delta \bar{y}\left(x^{\prime}, \cdot\right) p_{n}\left(x^{\prime}, \cdot\right) d x^{\prime}\right\|_{L^{2}(0, T)}^{2} \leq C, \forall n \geq 1 .
$$

This is implied by (2.15) and the fact that

$$
\bar{y}\left(x^{\prime}, t\right)=\sum_{j \geq 1} e^{-\lambda_{j} \int_{0}^{t} a\left(\int_{\Omega} \bar{y}(\xi, s) d \xi\right) d s}\left(\bar{y}_{0}, \phi_{j}\right) \phi_{j}\left(x^{\prime}\right),
$$

and

$$
p_{n}\left(x^{\prime}, t\right)=\sum_{j \geq 1} e^{-\lambda_{j} \int_{t}^{T} \alpha_{w_{n}}(s) d s}\left(\varphi_{n}^{T}, \phi_{j}\right) \phi_{j}\left(x^{\prime}\right) .
$$


Indeed, one has

$$
\begin{aligned}
& \left\|\beta_{w_{n}}(\cdot) \int_{\Omega} \Delta \bar{y}\left(x^{\prime}, \cdot\right) p_{n}\left(x^{\prime}, \cdot\right) d x^{\prime}\right\|_{L^{2}(0, T)}^{2} \\
& \quad=\int_{0}^{T}\left|\beta_{w_{n}}(t)\right|^{2}\left|\int_{\Omega} \Delta \bar{y}\left(x^{\prime}, t\right) p_{n}\left(x^{\prime}, t\right) d x^{\prime}\right|^{2} d t \\
& \quad \leq C \int_{0}^{T}\left|\sum_{j \geq 1} \lambda_{j} e^{-\lambda_{j} \int_{0}^{t} a\left(\int_{\Omega} \bar{y} d x^{\prime}\right) d s}\left(\bar{y}_{0}, \phi_{j}\right) e^{-\lambda_{j} \int_{t}^{T} \alpha_{w_{n}}(s) d s}\left(\varphi_{n}^{T}, \phi_{j}\right)\right|^{2} d t \\
& \quad \leq C \int_{0}^{T}\left(\sum_{j \geq 1} \lambda_{j}^{2} e^{-2 \lambda_{j} a_{0} t} e^{-2 \lambda_{j} a_{0}(T-t)} e^{2 R_{0} \sqrt{\lambda_{j}}}\left|\left(\bar{y}_{0}, \phi_{j}\right)\right|^{2}\right) d t\left\|\varphi_{n}^{T}\right\|_{\omega}^{2} \\
& \quad \leq C T\left\|\bar{y}_{0}\right\|^{2}\left(\sum_{j \geq 1} \lambda_{j}^{2} e^{-2 \lambda_{j} a_{0} T} e^{2 R_{0} \sqrt{\lambda_{j}}}\right)\left\|\varphi_{n}^{T}\right\|_{\omega}^{2} \\
& \leq C T\left\|\bar{y}_{0}\right\|^{2}<+\infty .
\end{aligned}
$$

Thanks to (2.20), it can be assumed that $w_{n}$ converges weakly in $L^{2}(Q)$ to a function $w,\left(w_{n}\right)_{t}$ converges weakly-* in $L^{\infty}\left(0, T ; L^{2}(\Omega)\right), \int_{\Omega} w_{n} d x$ converges strongly in $L^{2}(0, T)$ and $\zeta_{n}$ converges strongly in $L^{2}(Q)$ to the solution to $(2.13)$ Now, let us see that, at least for a subsequence, one has

$$
\varphi_{n} \rightarrow \varphi \text { weakly in } L^{2}(\Omega \times(0, T-\delta)) \forall \delta>0
$$

with

$$
\beta_{w}(t) \int_{\Omega} \Delta \bar{y}\left(x^{\prime}, t\right) \varphi\left(x^{\prime}, t\right) d x^{\prime}=0 \text { in }(0, T) .
$$

Indeed, the sequence $\left\{\varphi_{n}\right\}$ can be assumed to converge to some $\varphi$ weakly in $L^{2}(\Omega \times(0, T-\delta))$ for all $\delta>0$, since we have the estimates

$$
\begin{aligned}
\iint_{\Omega \times(0, T-\delta)}\left|\varphi_{n}\right|^{2} d x d t & \leq 2 \iint_{\Omega \times(0, T-\delta)}\left|p_{n}\right|^{2} d x d t+2 \iint_{\Omega \times(0, T-\delta)}\left|\zeta_{n}\right|^{2} d x d t \\
& \leq C_{\delta} \iint_{Q} e^{-2 s \sigma} \xi^{3}\left|p_{n}\right|^{2} d x d t+C \\
& \leq C_{\delta} C\left\|\varphi_{n}^{T}\right\|_{\omega}^{2}+C \\
& =C_{\delta} C+C .
\end{aligned}
$$

Here, we have used (2.14). Obviously, $\varphi$ solves in $Q$ the PDE

$$
-\varphi_{t}-\alpha_{w}(t) \Delta \varphi+\beta_{w}(t) \int_{\Omega} \Delta \bar{y}\left(x^{\prime}, t\right) \varphi\left(x^{\prime}, t\right) d x^{\prime}=0
$$

and $\varphi(x, t)=0$ in $\omega \times(0, T)$. In particular, writing this equation in $\omega \times(0, T)$, we deduce that (2.22) holds. An additional consequence is that $\varphi$ vanishes identically. 
From (2.20)-(2.22) and the fact that $\zeta_{n}$ is bounded in $L^{2}(Q)$, we see that

$$
\beta_{w_{n}}(\cdot) \int_{\Omega} \Delta \bar{y}\left(x^{\prime}, \cdot\right) \varphi_{n}\left(x^{\prime}, \cdot\right) d x^{\prime} \rightarrow 0 \text { weakly in } L^{2}\left(0, T ; H^{-1}(\Omega)\right)
$$

and, taking into account that $\zeta_{n}$ solves (2.13) for $p=p_{n}$ and $w=w_{n}$, we see that (2.19) holds.

Proof of (2.17). At this point, we will use the energy and observability estimates satisfied by $p$ and the energy estimates satisfied by $\zeta$.

From (2.12) and (2.14), we find that

$$
\|p(\cdot, 0)\|^{2} \leq C \int_{T / 4}^{3 T / 4}\|p(\cdot, t)\|^{2} d t \leq C\left\|\varphi^{T}\right\|_{\omega}^{2} .
$$

On the other hand, from (2.13) we have that

$$
\|\zeta(\cdot, 0)\|^{2} \leq C\left\|\beta_{w}(\cdot) \int_{\Omega} \Delta \bar{y}\left(x^{\prime}, \cdot\right) p\left(x^{\prime}, \cdot\right) d x^{\prime}\right\|_{L^{2}\left(0, T ; H^{-1}(\Omega)\right)}^{2}
$$

and, arguing as in (2.20), the following is found:

$$
\|\zeta(\cdot, 0)\|^{2} \leq C\left\|\varphi^{T}\right\|_{\omega}^{2}
$$

Finally, noting that $\varphi=p+\zeta$ and putting together (2.23) and (2.24), we get (2.10).

Now, let us prove the estimate (2.11). Taking into account the Carleman estimates satisfied by $p$ and arguing as in the proof of (2.20), we find:

$$
\begin{aligned}
I(\varphi) & \leq 2 I(p)+2 I(\zeta) \\
& \leq 2 I(p)+C\left(\|\zeta\|_{L^{2}\left(0, T ; H^{2}(\Omega)\right)}^{2}+\left\|\zeta_{t}\right\|_{L^{2}(Q)}^{2}\right) \\
& \leq C\left\|\varphi^{T}\right\|_{\omega}^{2}+C\left\|\beta_{w}(\cdot) \int_{\Omega} \Delta \bar{y}\left(x^{\prime}, \cdot\right) p\left(x^{\prime}, \cdot\right) d x^{\prime}\right\|_{L^{2}(0, T)}^{2} \\
& \leq C\left\|\varphi^{T}\right\|_{\omega}^{2}
\end{aligned}
$$

Hence, using (2.16), we deduce (2.11).

\subsection{Approximate Null Controllability of (1.7) with Controls Uniformly Bounded in $H^{1}\left(0, T ; L^{2}(\omega)\right)$}

The following holds:

Proposition 2.2. Let the assumptions (1.2) be satisfied and let us assume that $w \in Z$ and $\|w\|_{Z} \leq K$. For any $\delta>0$ and any $z_{0} \in L^{2}(\Omega)$, there exists a control $v_{\delta} \in$ $H^{1}\left(0, T ; L^{2}(\omega)\right.$ such that the corresponding solution $z$ to (1.7) satisfies

$$
\left\|z_{\delta}(\cdot, T)\right\| \leq \delta
$$


Moreover, the controls $v_{\delta}$ can be chosen such that

$$
\left\|v_{\delta}\right\|_{H^{1}\left(0, T ; L^{2}(\omega)\right)} \leq C\left\|z_{0}\right\|,
$$

with $C$ only depending on $\Omega, \omega, T, a_{0}, a_{1}, M$ and $K$.

Proof. For each $\delta>0$, let us consider the following functional on $L^{2}(\Omega)$ :

$$
F_{\delta}\left(\varphi^{T}\right):=\frac{1}{2} \iint_{\omega \times(0, T)} e^{-2 s \sigma} \xi^{3}|\varphi|^{2} d x d t+\delta\left\|\varphi^{T}\right\|+\left(z_{0}, \varphi(\cdot, 0)\right) .
$$

Here, for each $\varphi^{T} \in L^{2}(\Omega), \varphi$ is the corresponding solution of (1.7).

It is then clear that $F_{\delta}: L^{2}(\Omega) \mapsto \mathbb{R}$ is continuous and strictly convex. From (2.10), we also have that $F_{\delta}$ is coercive. Then, let $\varphi_{\delta}^{T}$ be the unique minimizer of $F_{\delta}$ and let us denote by $\varphi_{\delta}$ the associated solution to (1.7). Obviously, either $\varphi_{\delta}^{T}=0$ or

$$
\left\langle F_{\delta}^{\prime}\left(\varphi_{\delta}^{T}\right), \varphi^{T}\right\rangle=0 \quad \forall \varphi^{T} \in L^{2}(\Omega) .
$$

Suppose that $\varphi_{\delta}^{T} \neq 0$. Then, taking in (1.7)

$$
v=v_{\delta}:=\left.e^{-2 s \sigma} \xi^{3} \varphi_{\delta}\right|_{\omega \times(0, T)},
$$

denoting by $z_{\delta}$ the associated state and using $(2.27)$, we get

$$
\left(z_{\delta}(\cdot, T)+\frac{\delta}{\left\|\varphi_{\delta}^{T}\right\|} \varphi_{\delta}^{T}, \varphi^{T}\right)=0 \quad \forall \varphi^{T} \in L^{2}(\Omega)
$$

which implies

$$
\left\|z_{\delta}(\cdot, T)\right\|=\delta .
$$

In view of (2.10), we also have

$$
\iint_{\omega \times(0, T)} e^{2 s \sigma} \xi^{-3}\left|v_{\delta}\right|^{2} d x d t \leq C\left\|z_{0}\right\|^{2}
$$

for some $C=C\left(\Omega, T, a_{0}, a_{1}, M, K\right)$. This shows that, if $\varphi_{\delta}^{T} \neq 0$, we can find controls for which (2.25) and (2.29) hold. Obviously, this is also true if $\varphi_{\delta}^{T}=0$.

Next, we prove that the $v_{\delta, t}$ are uniformly bounded in $L^{2}(\omega \times(0, T))$. From the definition of $v_{\delta}$, we have $v_{\delta, t}=\left(e^{-2 s \sigma} \xi^{3}\right)_{t} \varphi_{\delta}+e^{-2 s \sigma} \xi^{3} \varphi_{\delta, t}$ in $\omega \times(0, T)$ and $v_{\delta, t}=0$ elsewhere. Hence, it is immediate that

$$
\left\|v_{\delta, t}\right\|_{L^{2}(\omega \times(0, T))}^{2} \leq C\left(\iint_{Q} e^{-2 s \sigma} \xi^{3}\left|\varphi_{\delta}\right|^{2} d x d t+\iint_{Q} e^{-2 s \sigma} \xi^{-1}\left|\varphi_{\delta, t}\right|^{2} d x d t\right) .
$$

Recalling the estimates (2.11) and (2.29), we deduce that

$$
\left\|v_{\delta, t}\right\|_{L^{2}(\omega \times(0, T))} \leq C\left\|z_{0}\right\|,
$$

as desired.

This ends the proof. 


\section{Proof of Theorem 1.1}

This section is devoted to prove the local exact controllability to the trajectories result in Theorem 1.1.

\subsection{Local Approximate Null Controllability of (1.1) with Con- trols Uniformly Bounded in $L^{2}(\omega \times(0, T))$}

We will prove that (1.1) is locally approximately null-controllable, with controls uniformly bounded in $L^{2}(\omega \times(0, T))$. Thus, let us introduce the Banach spaces $G$ and $W$, with

$$
\begin{aligned}
& G:=\left\{(v, w): v, v_{t} \in L^{2}\left(0, T ; L^{2}(\omega)\right), w, w_{t} \in L^{\infty}\left(0, T ; H_{0}^{1}(\Omega)\right),\right. \\
&\left.w_{t t} \in L^{2}\left(0, T ; H^{-1}(\Omega)\right)\right\}, \\
& W:=\left\{w: w, w_{t} \in L^{\infty}\left(0, T ; H_{0}^{1}(\Omega)\right), w_{t t} \in L^{2}\left(0, T ; H^{-1}(\Omega)\right)\right\} .
\end{aligned}
$$

Let $K>0$ and $w \in Z$ be given, with $\|w\|_{Z} \leq K$. For each $\delta>0$, let us consider a control $v_{\delta}$ furnished by Proposition 2.2 and let us denote by $z_{\delta}$ the solution to (1.7) associated to $v_{\delta}$. From (2.25) and (2.26), one has:

$$
\begin{gathered}
\left\|v_{\delta}\right\|_{H^{1}\left(0, T ; L^{2}(\omega)\right)} \leq C(K)\left\|z_{0}\right\|, \\
\left\|z_{\delta}\right\|_{Z} \leq C(K)\left\|z_{0}\right\|, \quad\left\|z_{\delta}(\cdot, T)\right\| \leq \delta
\end{gathered}
$$

and

$$
\left\|z_{\delta}\right\|_{W} \leq C_{\delta}(K)\left\|z_{0}\right\|_{H^{2}},
$$

where we have omitted the dependence of the constants on $\Omega, \omega, T, a_{0}, a_{1}$ and $M$.

Let us introduce the mapping $\Lambda_{\delta}: B_{Z}(K) \mapsto 2^{Z}$, where $B_{Z}(K)$ is the closed ball in $Z$ of radius $K$ centered at 0 and

$$
\begin{gathered}
\Lambda_{\delta}(w)=\left\{z_{\delta} \in Z:\left(v_{\delta}, z_{\delta}\right) \text { is a control-state pair in } G,\right. \\
(1.7) \text { and }(3.31)-(3.33) \text { hold }\} .
\end{gathered}
$$

Then, if $\left\|z_{0}\right\|_{H^{2}(\Omega)}$ is sufficiently small, the multi-valued mapping $\Lambda_{\delta}$ satisfies the hypotheses of Kakutani's Fixed-Point Theorem. Indeed, the following holds:

- $\Lambda_{\delta}$ is well defined; also for each $w \in Z, \Lambda_{\delta}(w)$ is non-empty and convex (a consequence of Proposition 2.2).

- There exists $\epsilon>0$ (depending on $\Omega, \omega, T, a_{0}, a_{1}, M$ and $K$, but independent of $\delta$ ) such that, if $\left\|z_{0}\right\|_{H^{2}} \leq \epsilon$, one has $\Lambda_{\delta}(w) \subset B_{Z}(K)$ for all $w \in B_{Z}(K)$. This is a consequence of (3.32).

- There exist a compact set $W_{\delta} \subset B_{Z}(K)$ such that, whenever $\left\|z_{0}\right\|_{H^{2}} \leq \epsilon$ and $w \in B_{Z}(K)$, one has $\Lambda_{\delta}(w) \subset W_{\delta}$. This is a consequence of the fact that $W \hookrightarrow Z$ with a compact embedding. 
- $\Lambda_{\delta}$ has a closed graph in $Z$. This is not difficult to check. Indeed, let the $w_{n}$ be given, with $w_{n} \rightarrow w$ strongly in $Z$, let us assume that the $z_{n} \in \Lambda_{\delta}\left(w_{n}\right)$ and $z_{n} \rightarrow z$ strongly in $Z$. Then, there exist controls $v_{n, \delta}$ such that, for all $n \geq 1$, the couple $\left(v_{n, \delta}, z_{n}\right)$ belongs to $G$ and satisfies (1.7) and (3.31)(3.33) for all $n \geq 1$.

We can take limits in (1.7) and get

$$
\begin{cases}z_{t}-\alpha_{w}(t) \Delta z+\beta_{w}(t)\left(\int_{\Omega} z\left(x^{\prime}, t\right) d x^{\prime}\right) \Delta \bar{y}=v 1_{\omega} & \text { in } Q \\ z(x, t)=0 & \text { on } \Sigma, \\ z(x, 0)=z_{0}(x) & \text { in } \Omega .\end{cases}
$$

Consequently, one has $z \in \Lambda_{\delta}(w)$.

Therefore, if $\left\|z_{0}\right\|_{H^{2}} \leq \epsilon\left(\Omega, \omega, T, a_{0}, a_{1}, M, K\right), \Lambda_{\delta}$ possesses at least one fixed point $z_{\delta}$. Obviously, $z_{\delta}$ is a solution to (1.1) associated to a control $v_{\delta}$ such that (3.31)-(3.33) holds.

This ends the proof.

\subsection{Passage to the limit}

We know that, for any small $\delta>0$, there exist couples $\left(z_{\delta}, v_{\delta}\right)$ with $z_{\delta} \in$ $B_{Z}(K)$ and $v_{\delta} \in H^{1}\left(0, T ; L^{2}(\omega)\right)$, that satisfy the state equation

$$
\begin{cases}z_{\delta, t}-a\left(\int_{\Omega}\left(z_{\delta}+\bar{y}\right) d x^{\prime}\right) \Delta z_{\delta}-m\left(z_{\delta}\right) \Delta \bar{y}=v_{\delta} 1_{\omega} & \text { in } Q, \\ z_{\delta}(x, t)=0 & \text { on } \Sigma, \\ z_{\delta}(x, 0)=z_{0}(x) & \text { in } \Omega,\end{cases}
$$

where

$$
m\left(z_{\delta}\right)=a\left(\int_{\Omega}\left(z_{\delta}+\bar{y}\right)\left(x^{\prime}, t\right) d x^{\prime}\right)-a\left(\int_{\Omega} \bar{y}\left(x^{\prime}, t\right) d x^{\prime}\right)
$$

and also (3.31)-(3.32).

We can take limits in the nonlinear system (3.34) as $\delta \rightarrow 0$. Indeed, there exist subsequences of $\left\{z_{\delta}\right\}$ and $\left\{v_{\delta}\right\}$, again indexed by $\delta$, such that

$$
\left\{\begin{array}{lll}
z_{\delta} \rightarrow z & \text { weakly in } & L^{2}\left(0, T ; H^{2}(\Omega)\right) \\
z_{\delta, t} \rightarrow z_{t} & \text { weakly in } & L^{2}(Q) \\
z_{\delta, t} \rightarrow z_{t} & \text { strongly in } & L^{2}\left(0, T ; H_{0}^{1}(\Omega)\right) \\
v_{\delta} \rightarrow v & \text { weakly in } & L^{2}(\omega \times(0, T))
\end{array}\right.
$$

and

$$
z_{\delta}(\cdot, T) \rightarrow z(\cdot, T) \quad \text { strongly in } \quad L^{2}(\Omega) .
$$

Therefore, it is clear that the functions $z$ and $v$ satisfy (1.6) and, moreover,

$$
z(x, T)=0 \quad \text { in } \Omega .
$$

The proof of Theorem 1.1 is thus complete. 


\section{Some Additional Comments and Questions}

\subsection{Boundary Controllability}

In this subsection, we deal with the boundary local exact controllability to the trajectories of nonlinear system (1.1). We assume that $\Omega$ is an interval $(0, L)$, which we will denote by $I$ and the controls act on the whole boundary. If we set $y=z+\bar{y}$ and $y_{0}=z_{0}+\bar{y}_{0}$, we obtain

$$
\begin{cases}z_{t}-a\left(\int_{I}(z+\bar{y}) d x^{\prime}\right) z_{x x}-m(z) \bar{y}_{x x}=0 & \text { in } I \times(0, T), \\ z(0, t)=v_{0}(t), z(L, t)=v_{1}(t) & \text { on }(0, T), \\ z(x, 0)=z_{0}(x) & \text { in } I .\end{cases}
$$

Thus, the boundary local exact controllability to the trajectories to $\bar{y}$ is equivalent to the boundary local null controllability of the solution to (4.37). For the proof of this property, we proceed by steps:

STEP 1: Let us set $\widetilde{Z}:=C^{1,1}(\bar{Q})$. Then, for any $w \in \widetilde{Z}$, we prove boundary observability estimates for the solutions to (1.8), similar to (2.10) and (2.11).

The proof is as in Proposition 2.1. The crucial point is to prove that, if the adjoint states $\varphi_{n}$ satisfy $\varphi_{n} \rightarrow \varphi$ weakly in $L^{2}(I \times(0, T-\delta))$ and $\varphi_{x}(0, t)=$ $\varphi_{x}(L, t)=0$ for all $t \in(0, T-\delta)$, then $\varphi=0$ in $I \times(0, T-\delta)$ (here, $\delta>0$ is arbitrarily small).

STEP 2: Using these inequalities and following the ideas in [13], we can get controls $v_{0}$ and $v_{1}$ belonging to $C^{1,1 / 4}([0, T])$ such that

$$
\left\|v_{0}\right\|_{C^{1,1 / 4}[0, T]}+\left\|v_{1}\right\|_{C^{1,1 / 4}[0, T]} \leq C\left\|z_{0}\right\|
$$

and, furthermore, the solution $z$ to the associated linearized system satisfies:

$$
z(\cdot, T)=0 \text { in } I .
$$

where $C$ only depend of $I, a_{0}, a_{1}$ and $\|w\|_{\tilde{Z}}$.

STEP 3: As a consequence, the boundary local null controllability of (4.37) can be established. Again, the argument relies on a previous result concerning local approximate controllability with uniform estimates. We omit the details, in view of the similarity to the distributed control case.

Remark 4.1. At present, we have not been able to establish a result of this kind in higher dimensions. Some (maybe technical) difficulties are found in the proof of the required boundary observability estimates.

\subsection{Other Nonlinear Control Problems}

The controllability to the trajectories of the system 


$$
\begin{cases}y_{t}-a\left(\int_{\Omega} y\left(x^{\prime}, t\right) d x^{\prime}\right) \Delta y+b(x, t) y=v 1_{\omega} & \text { in } Q \\ y(x, t)=0 & \text { on } \Sigma, \\ y(x, 0)=y_{0}(x) & \text { in } \Omega,\end{cases}
$$

is an open question.

If we try to apply the same techniques, a difficulty is found in the proof of the needed observability inequality.

More precisely, if we argue as in the proof of the Proposition 2.1, we must set

$$
\varphi=p+\zeta
$$

with $p$ and $\zeta$ respectively satisfying (2.12) and

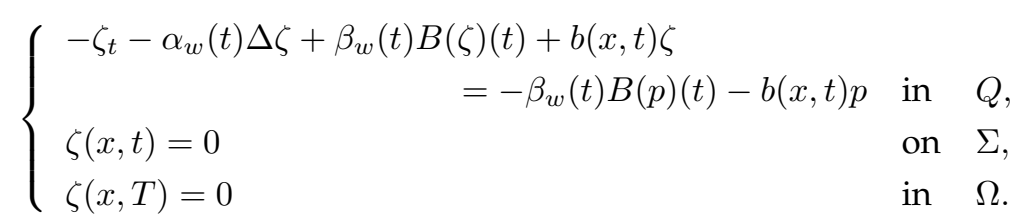

Unfortunately, it is not clear whether (2.16) holds in this case; indeed, in view of the presence of the term $-b(x, t) p$ in (4.41), it seems difficult to establish (2.19) in the related contradiction argument.

In fact, we are able to prove a result similar to Proposition 2.1 only when $b=b(x)$ or $b=b(t)$. In other words:

- If $b:=b(x)$, working with $A z=\left(\alpha_{w}(t) \Delta-b(x)\right) z$ instead of $\Delta z$ in (1.7) and (1.8), we can get result similar to Proposition 2.1 and then Theorem 1.1 .

- If $b:=b(t)$, we can introduce the change of variables $\tilde{y}=e^{\int_{0}^{t} b(s) d s} y$ and, then, deduce the results in Section 2 and Section 3.

\section{Appendix: Proof of Lemma 2.2}

Let $w \in Z$ be given and let us set

$$
\alpha(t):=a\left(\int_{\Omega} w\left(x^{\prime}, t\right) d x^{\prime}\right) .
$$

Let us assume that $\varphi$ satisfies

$$
\begin{cases}-\varphi_{t}-\alpha(t) \Delta \varphi=0 & \text { in } \quad Q \\ \varphi=0 & \text { on } \Sigma, \\ \varphi(x, T)=f(x) & \text { in } \quad \Omega .\end{cases}
$$


From the standard Carleman estimates for $\varphi$, one has

$$
\iint_{Q} e^{-C_{0} /(T-t)}|\varphi|^{2} d x d t \leq \tilde{C}_{0} \iint_{\omega \times(0, T)} e^{-2 s \sigma} \xi^{3}|\varphi|^{2} d x d t
$$

where $C_{0}$ only depends on $\Omega, \omega, T, a_{0}, a_{1} . M$ and $\|w\|_{Z}$ and $\tilde{C}_{0}$ only depends on $\Omega, \omega, a_{0}, a_{1}, M$ and $\|w\|_{Z}$, see [9] and [7]. Taking into account that

$$
\varphi(x, t)=\sum_{j \geq 1} e^{-\lambda_{j} \int_{t}^{T} \alpha(s) d s}\left(f, \phi_{j}\right) \phi_{j}
$$

and

$$
\|\varphi\|^{2}=\sum_{j \geq 1} e^{-2 \lambda_{j} \int_{t}^{T} \alpha(s) d s}\left|\left(f, \phi_{j}\right)\right|^{2},
$$

we see from (5.42) that

$$
\int_{0}^{T} \sum_{j \geq 1} e^{-2 \lambda_{j} \int_{t}^{T} \alpha(s) d s-C_{0} /(T-t)}\left|\left(f, \phi_{j}\right)\right|^{2} d t \leq \tilde{C}_{0} \iint_{\omega \times(0, T)} e^{-2 s \sigma} \xi^{3}|\varphi|^{2} d x d t
$$

and, consequently,

$\sum_{j \geq 1}\left(\int_{0}^{T} e^{-2 \lambda_{j} a_{1}(T-t)-C_{0} /(T-t)} d t\right)\left|\left(f, \phi_{j}\right)\right|^{2} \leq \tilde{C}_{0} \iint_{\omega \times(0, T)} e^{-2 s \sigma} \xi^{3}|\varphi|^{2} d x d t$.

The asymptotic behavior of the integrals in the left hand side is well known. Indeed, one has

$$
\int_{0}^{T} e^{-2 \lambda a_{1}(T-t)-C_{0} /(T-t)} d t \sim\left(\frac{\pi^{2} C_{0}}{4\left(\lambda a_{1}\right)^{3}}\right)^{1 / 4} e^{-4 \sqrt{C_{0} a_{1} \lambda}}, \text { as } \lambda a_{1} \rightarrow \infty
$$

(see for instance [8]). Thus, there exists $\tilde{C}_{1}$, again depending on $\Omega, \omega, a_{0}, a_{1}, M$ and $\|w\|_{Z}$, such that

$$
\int_{0}^{T} e^{-2 \lambda_{j} a_{1}(T-t)-C_{0} /(T-t)} d t \geq \tilde{C}_{1} e^{-2 R_{0} \sqrt{\lambda_{j}}} \quad \forall j \geq 1 .
$$

Obviously, this leads to (2.9) and ends the proof.

\section{References}

[1] V. M. Alekseev, V. M. Tikhomorov, S. V. Formin, Optimal Control, Consultants Bureau, New York, 1987.

[2] N. H. Chang, M. Chipot, On some model diffusion problems with a nonlocal lower ordem, Chin. Ann. Math. 24 (B:2) (2003) 147-166. 
[3] N. H. Chang, M. Chipot, Nonlinear Nonlocal Evolution Problems, RACSAM, Rev. R. Acad. Cien. Serie A. Mat, Vol 97 (3), (2003), p. 393-415.

[4] H.R. Clark, E. Fernández-Cara, J. Limaco, L.A. Medeiros, Theoretical and numerical local null controllability for a parabolic system with local and nonlocal nonlinearities, Applied Mathematics and Computation 223 (2013) 483-505.

[5] C. Fabre, J.P. Puel, E. Zuazua, Approximate controllability of the semilinear heat equation, Proc. Roy. Soc. Edinburgh Sect. 125A (1995) 31-61,

[6] E. Fernández-Cara, Q. Lü and E. Zuazua, Null controllability of linear heat and wave equations with nonlocal spatial terms, SIAM, J. Control Optim. 54(4), (2016) 2009-2019.

[7] E. Fernández-Cara, E. Zuazua, Null and approximate controllability for weakly blowing up semilinear heat equations, Ann. Inst. Henri Poincaré, Analyse non linéaire 17, 5 (2000) 583-616.

[8] E. Fernández-Cara and E. Zuazua, The cost of approximate controllability for heat equations: The linear case, Adv. Differential Equations, 5 (2000), pp. 465-514.

[9] A.V. Fursikov, O.Yu. Imanuvilov, Controllability of Evolution Equations, Lecture Notes Series, vol. 34, Seoul National University, Research Institute of Mathematics, Global Analysis Research Center, Seoul, 1996.

[10] O. Yu. Imanuvilov, M. Yamamoto, Carleman inequalities for parabolic equations in Sobolev spaces of negative order and exact controllability for semilinear parabolic equations, Publ. Res. Inst. Math. Sci. 39 (2) (2003) 227-274.

[11] O. Yu. Imanuvilov, Controllability of parabolic equations, Mat. Sb. 186 (1995), pp. 879-900.

[12] O. A. Ladyzhenskaya, V. A. Solonnikov, N. N. Ural'ceva, Linear and Quasilinear Equations of Parabolic Type, Transl, Math. Monogr. 23, AMS, Providence, RI, 1968.

[13] $\mathrm{Xu} \mathrm{Liu,} \mathrm{Xu}$ Zhang, Local controllability of multidimensional quasi-linear parabolic equations SIAM J. Control Optimiz. Vol. 50, No. 4, pp. 2046-2064. 